\title{
Chemotherapy with trifluridine/tipiracil prolongs progression-free survival time and improves quality of life. A case report of 59-year-old woman with metastatic rectal cancer
}

\section{Beata Kania-Zembaczyńska}

Oncology and Hematology Department,

Beskid Oncology Centre - John Paul II Memorial City Hospital in Bielsko-Biala

Correspondence: Beata Kania-Zembaczyńska Department of Oncology and Hematology, Beskid Oncology Centre - John Paul II Memorial City Hospital in Bielsko-Biala 43-300 Bielsko-Biała, ul. Wyzwolenia 18 phone: 334984000

Received:

12.03.2020

Accepted:

31.03.2020

DOI: 10.24292/01.OR.120310320

Copyright $\odot$ Medical Education. All rights reserved.

\section{ABSTRACT}

The paper presents a clinical case of a patient with a long-term response to IV line chemotherapy with trifluridine/tipiracil. The treatment allowed to maintain a good quality of life with acceptable side effects.

Key words: rectal cancer, chemotherapy, trifluridine/tipiracil, progression-free survival, quality of life 


\section{INTRODUCTION}

Trifluridine/tipiracil (Lonsurf ${ }^{\oplus}$, TAS-102, FTD/TPI) is a complex orally administered antimetabolite which is an option for treatment of advanced metastatic colorectal cancer ( $\mathrm{mCRC}$ ) approved by European Medicines Agency (EMA) in patients who are refractory, or are not considered candidates for currently available therapies. The drug combines trifluridine, a thymidine-based nucleoside analogue, and tipiracil, which improves the bioavailability of trifluridine by inhibiting the enzyme thymidine phosphorylase, which is involved in its catabolism [1, 2]. In the randomized global phase III clinical trial RECOURSE, FTD/TPI prolonged overall survival (OS) and progression free survival (PFS) compared to placebo with a favorable safety profile [3].

\section{CASE REPORT}

The case report concerns a 59-year-old patient with rectal cancer diagnosed in 2003. In October 2003, abdominal-perineal amputation of the rectum was performed in the General Surgery Department of the Hospital in Oświęcim. In the postoperative examination, G2, pT2NOMx adenocarcinoma according to Astler-Coller B1 was found. Additionally there were 17 lymph nodes free of metastases and cancer cell clots in the lymph vessels. After the surgery the patient was qualified for complementary radiotherapy. From December $16^{\text {th }}, 2003$ until January $23^{\text {th }}$, 2013, complementary radiotherapy to total dose (Dc) $50.4 \mathrm{~Gy} / \mathrm{g}$ with concurrent leucovorin ( $\mathrm{FL}$ ) chemotherapy was performed. After radiotherapy, the patient underwent six cycles of chemotherapy. The patient was under control in the oncological outpatient clinic. In February 2007, a patient presented an episode of vaginal discharge with blood. In August 2007, the histopathological findings of vagina revealed adenocarcinoma. The patient was qualified for palliative chemotherapy CLF1. After administration of six cycles, the patient exhibited the stabilization, without further vaginal lesions confirmed by diagnostic imaging.

In April 2008, due to progression, anti-hemorrhagic brachytherapy of vaginal infiltration was performed at the Oncology Institute in Gliwice to Dc 42 Gy/g. After completing brachytherapy, 11 cycles of chemotherapy based on capecitabine were administered (the decision to continue treatment with capecitabine resulted from preventing excluding the patient options for further treatment in the drug program).

In March 2011, another cancer recurrence in the pelvic was diagnosed in PET/CT examination (CT scan result was inconclusive). Due to confined pelvic recurrence, the patient was qualified for surgery. The operation was performed in July 2011 at the Onco- logical and Reconstructive Surgery Clinic of the Oncology Institute in Gliwice - the recurrence in the lesser pelvis with the uterus, appendages and part of the vagina was removed. The patient remained under observation and treatment at the Oncology Institute in Gliwice. After one year, imaging examinations revealed lesions in the rectal cavity which were further confirmed by PET/ $\mathrm{CT}$ as a tumor in the pelvis in the rectal cavity. The patient was qualified for stereotactic radiotherapy for recurrence in the presacral area - three fractions of 7 Gy/tumor. In 2013 cystoscopy was performed due to hematuria and thickening of the bladder wall described in CT. Bladder infiltration was not confirmed at that time. Another cystoscopy with specimen collection in May 2014 confirmed the infiltration of adenocarcinoma that could correspond to recurrence of colorectal cancer. In imaging tests performed in December 2014, several metastatic lesions in the lungs up to $15 \mathrm{~mm}$ were described. The patient was referred for palliative chemotherapy to the Oncology and Hematooncology Department in Bielsko-Biala.

The patient was qualified for FOLFOX chemo-immunotherapy with bevacizumab as part of the NFZ drug program. Treatment began in March 2015. After two cycles, treatment with bevacizumab was terminated due to the appearance of a vesicocutaneous fistula in the sacral region, and therapy based on FOLFOX chemotherapy was continued. In June 2015, due to right-sided hydronephrosis as a result of ureter stenosis after radiotherapy, a nephrostomy was performed.

In total, 16 cycles of chemotherapy were given until January 2016. The treatment used, allowed to achieve regression of metastatic changes in the lungs according to RECIST 1.1.

Since June 2016, there was another urine leakage through the vesicocutaneous fistula in the sacrum area. Imaging studies described the progression of pelvic recurrence and metastasis in the lungs. Due to the infiltration of the bladder and ureters with secondary stagnation in the kidneys, the patient underwent second nephrostomy and surgical consultation.

The patient was operated in November 2016 in the Department of Urology and Urological Oncology in Bielsko-Biała. Palliative pelvic tumor resection was performed with removal of the bladder and bilateral ureterocutaneostomy, segmental resection of two loops of small intestine with the end-to-end anastomosis. The postoperative course was complicated by the occurrence of the caecal fistula due to which the patient was reoperated and the postoperative wound dehiscence. 
In January 2017, imaging studies described residual infiltration in the presacral area, partial intestinal obstruction, hepatic metastases, and progression of lung metastases.

The patient's condition after surgery improved, the patient did not show any clinical signs of gastrointestinal obstruction, stabilization of body weight was obtained (weight loss of approx. $10 \mathrm{~kg}$ in the perioperative and postoperative period).

No genetic mutations were found in the KRAS, NRAS and BRAF genes. The patient was qualified for anti-EGFR treatment as part

\section{FIGURE 1.}

Left paravertebral lymph nodes.

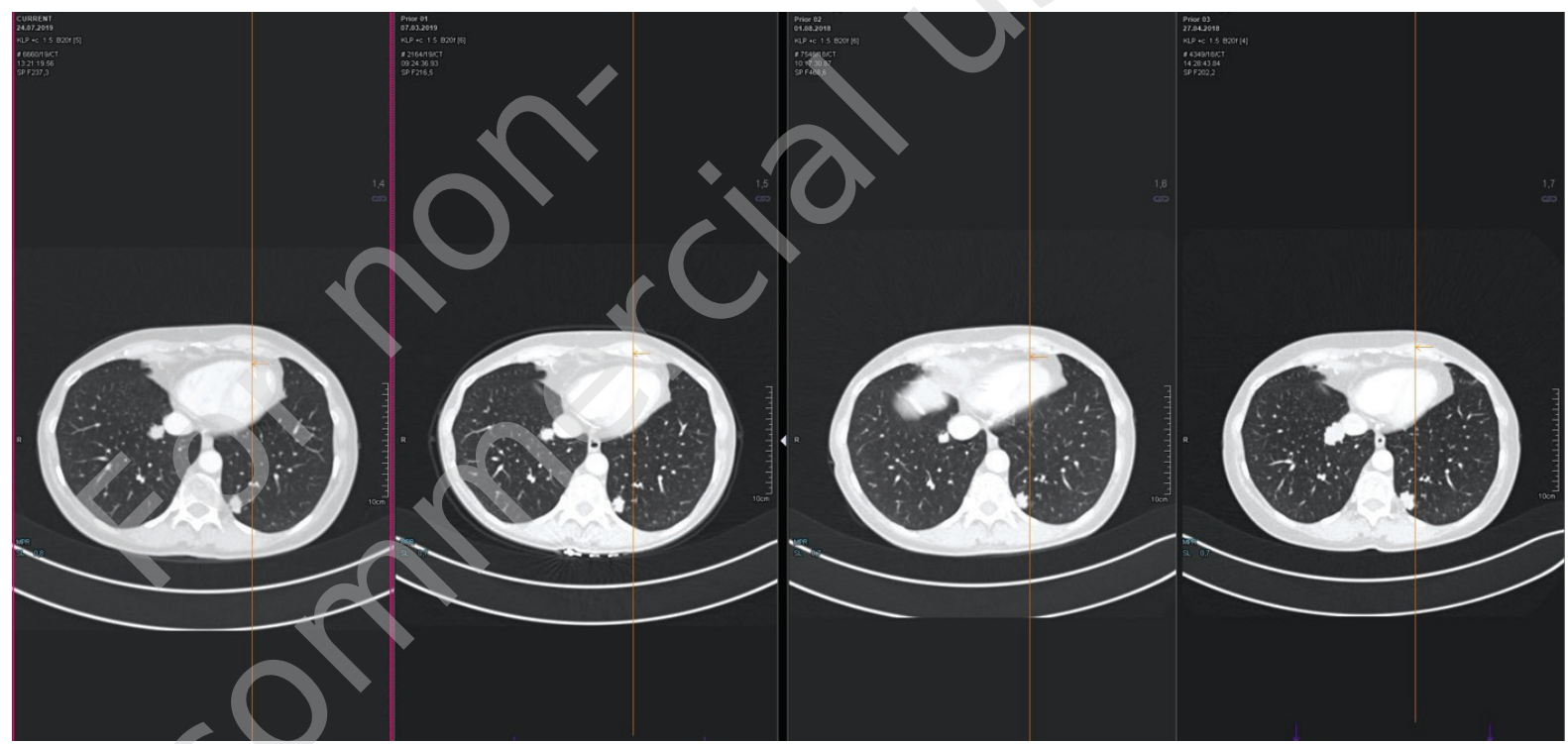

FIGURE 2.

Left lung - segment III.

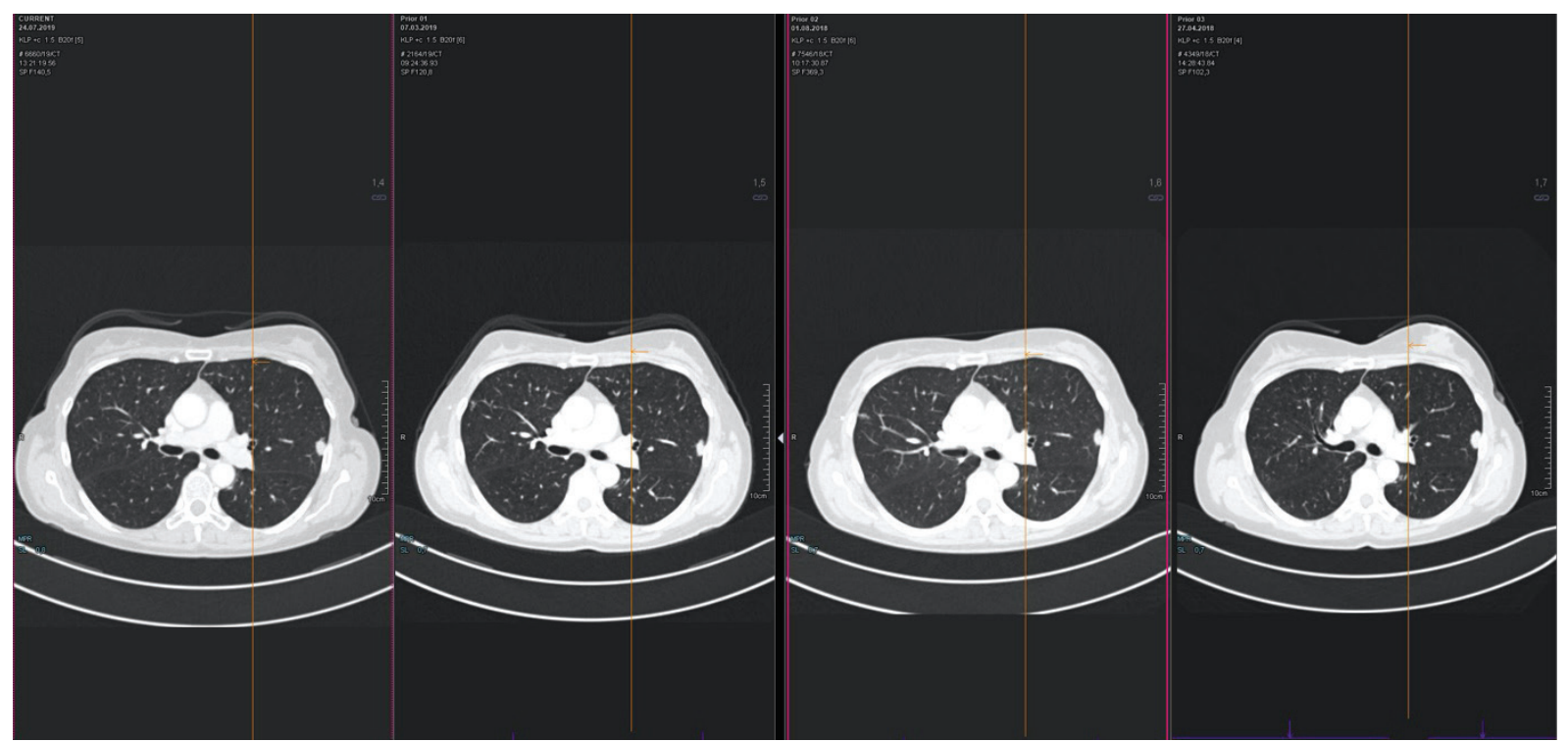




\section{FIGURE 3.}

Right lung - segment IV.

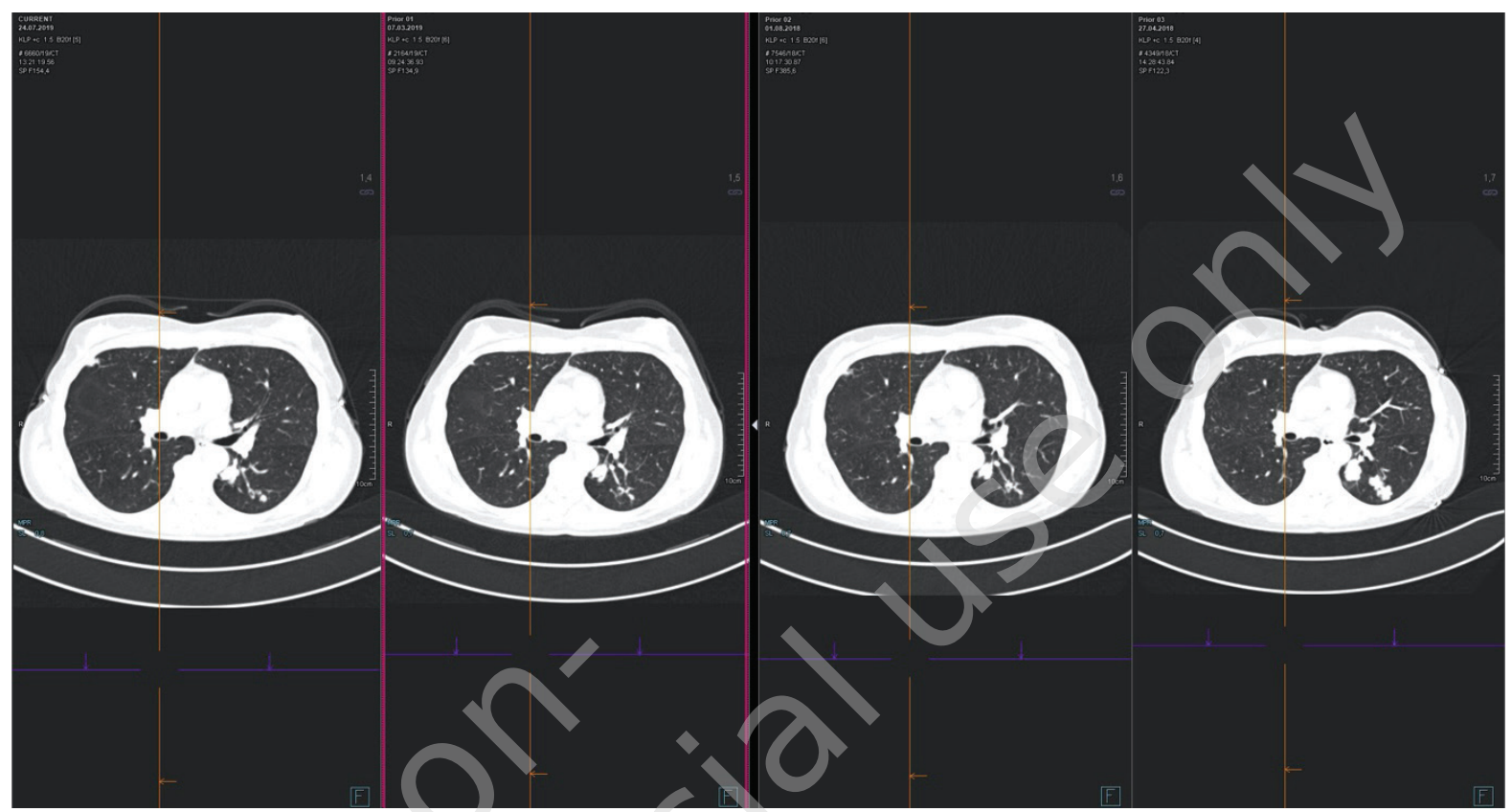

FIGURE 4.

Right lung - segment VIII.

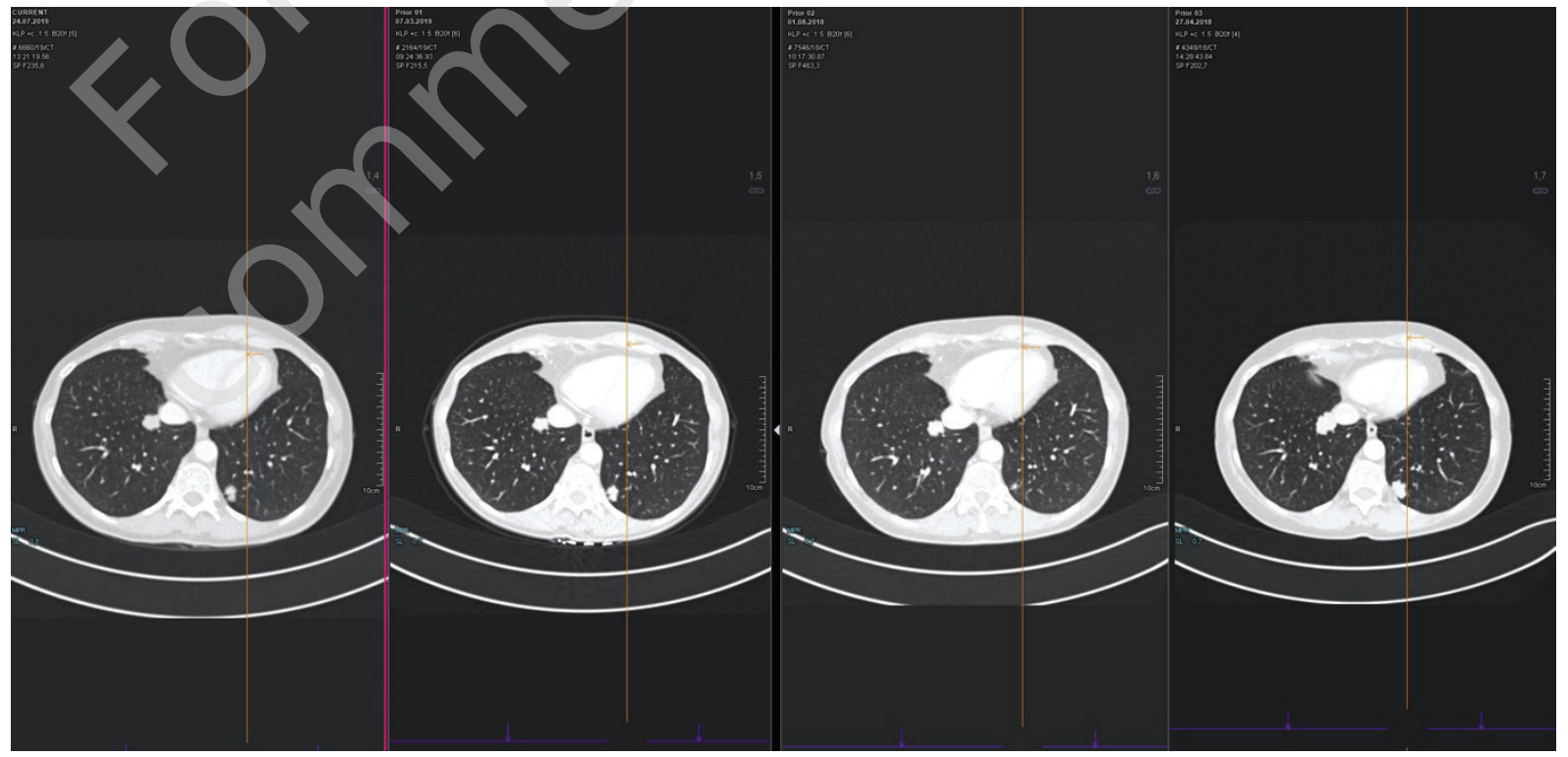

From May 2018, TAS-102 treatment was started at a dose of $2 \times$ $35 \mathrm{mg} / \mathrm{m}^{2} / 24 \mathrm{~h}$ financed by the patient and based on the donations from Servier in the fourth treatment line. At the same time, an application for financing treatment was submitted as part of emergency access to medical technology RDTL. The application was approved by the Voivodeship Consultant of Clinical Oncology, but was not approved by the Ministry of Health. The applica- tion was reissued after confirmation of the partial remission of the disease in control imaging performed after three months of treatment, which also received a negative response.

Radiological assessment of metastatic changes in the lungs (from the right: April 2018, August 2018, March 2019 and July 2019 study) (fig. 1-4). 
Up until October $31^{\text {st }}, 2019,16$ cycles of TAS-102 were given. Asymptomatic CTCAE - three neutropenia after the III cycle was observed during treatment due to which the dose was reduced to $2 \times 30 \mathrm{mg} / \mathrm{m}^{2} / 24 \mathrm{~h}$ in accordance with the SPC. The next dose reduction was done after the $\mathrm{V}$ cycle due to grade III neutropenia. The patient continues treatment at a dose of $2 \times 25 \mathrm{mg} / \mathrm{m}^{2} / 24 \mathrm{~h}$ During therapy, no other hematological toxicity was observed. The patient remains in good condition and is fully active.

The next radiological assessment is scheduled for December $20^{\text {th }}$, 2019. If the response to treatment persists, the patient will be qualified for treatment as part of the NFZ drug program.

\section{DISCUSSION}

The main aims of treatment in $\mathrm{MCRC}$ are to prolong OS and maintain quality of life (QoL) of the patient [4]. Although as shown in the analysis of RECOURSE the therapy with the trifluridine/ tipiracil chemotherapy cause some adverse effects, they are re- versible and predictable, and they did not affect the QoL. AEs are usually classified as mild or moderate, and can be managed by dose reductions or treatment delay. The analysis showed that the trifluridine/tipiracil treatment does not result in a deterioration of patient QoL versus placebo [5].

\section{CONCLUSION}

The case described demonstrates the possibility of obtaining a long-term response to TAS-102 in patients who have been treated while maintaining a good quality of life and acceptable toxicity.

Thanks to the financing of TAS-102 treatment as part of the NFZ drug program from November 2019, a larger group of patients remaining in good general condition will have the opportunity to receive therapy in the third and fourth lines in recurrent and refractory colorectal cancer.

\section{References}

1. European Medicines Agency. European public assessment report (EPAR) for Lonsurf. 2016. http://www.ema.europa.eu.

2. Lenz HJ, Stintzing S, Loupakis F. TAS-102, a novel antitumor agent: a review of the mechanism of action. Cancer Treatment Rev. 2015; 41: 777-83.

3. Mayer R, Van Cutsem E, Falcone A et al.; RECOURSE Study Group. Randomized trial of TAS-102 for refractory metastatic colorectal cancer. N Engl J Med. 2015; 372: 1909-19.

4. Funaioli C, Longobardi C, Martoni AA. The impact of chemotherapy on overall survival and quality of life of patients with metastatic colorectal cancer: a review of phase III trials. J Chemother. 2008; 20: 14-27.

5. Van Cutsem E, Falcone A, Garcia-Carbonero R et al. Proxies of quality of life in metastatic colorectal cancer: analyses in the RECOURSE trial. ESMO Open. 2017; 2(5): e000261. 